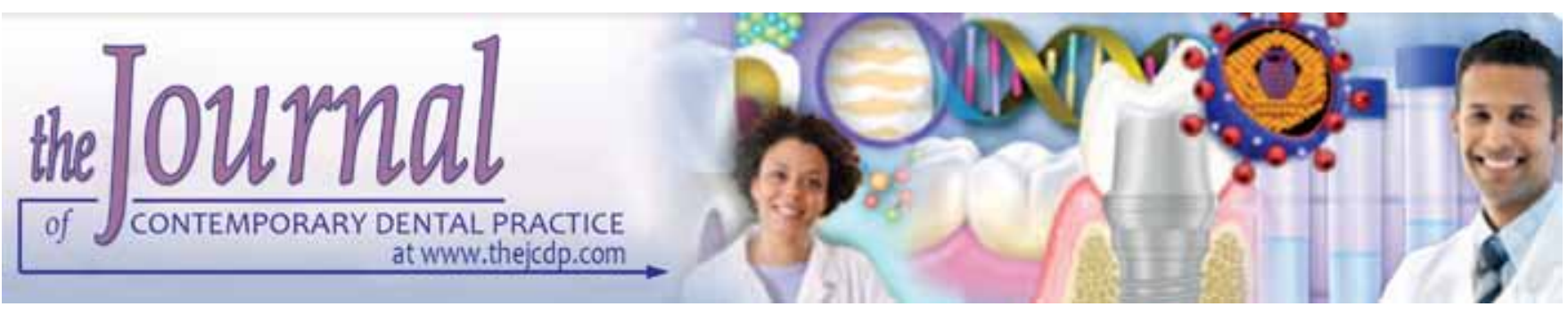

\title{
Effects of Bimax and Segmental Surgeries for Correction of Bimaxillary Dentoalveolar Protrusion Class I on Soft Tissue Parameters: Upper lip Thickness and Curvature, Nasolabial Angle and Nasal Prominence
}

\author{
Amin Rahpeyma, Saeedeh Khajehahmadi
}

\begin{abstract}
Aim: Results of this study can show if bimax surgery for posterior repositioning of maxilla and correction of BPCLI has priority to the currently used segmental orthognathic surgery or not.
\end{abstract}

Materials and methods: This study was done on 40 whiteskinned Iranian patients with bimaxillary dentoalveolar protrusion class I (BPCLI) who sought treatment for their deformity. In the first group, treatment includes segmental surgery for backward replacement of anterior segment of the upper and lower jaw. In the second group, treatment was bimax surgery, in which whole upper and lower jaw moved backward. Twenty patients were included in each group. For this purpose, we measured upper lip thickness (ULT, distance between LS and IA), nose prominence (NP, distance between nasal tip and the perpendicular line from upper lip vermilion on FHP), subsulcus depth (SSD, distance of SLS from this perpendicular line), $\mathrm{SN}$ to $\mathrm{H}$ line distance and finally, nasolabial angle (NLA) before and after surgery.

Results: In our study, $65 \%$ of patients were female and the mean of age was 27 (17-39) years old. The mean of SNA, SNB, ANB and INA in our patients were $81.7 \pm 2.9,78.8 \pm 2.8,4.50 \pm 1.4$ and $120 \pm 8.7$, respectively. All variables except SSD were analyzed with t-test to compare the results of two methods of surgery. Differences in the values of NP, NLA, SN to $\mathrm{H}$ line distance and ULT before and after segmental and bimax surgeries between before and after surgery were significant. After surgeries, ULT and the $\mathrm{SN}$ to $\mathrm{H}$ line distance reduced significantly, and NLA became corrected to its normal range (90-110).

Conclusion: The results of this study showed that bimax and segmental surgeries can effectively correct BPCLI. Because of possible dental and periodontal complications of segmental surgery, we highly recommend bimax surgery for treatment of BPCLI.

Keywords: Segmental surgery, Orthognathic surgery, Protrusion.

How to cite this article: Rahpeyma A, Khajehahmadi S. Effects of Bimax and Segmental Surgeries for Correction of Bimaxillary
Dentoalveolar Protrusion Class I on Soft Tissue Parameters: Upper lip Thickness and Curvature, Nasolabial Angle and Nasal Prominence. J Contemp Dent Pract 2013;14(6):1087-1093.

Source of support: Nil

Conflict of interest: None declared

\section{INTRODUCTION}

Bimaxillary protrusion (BP) is a dentofacial deformity which is defined by the protrusion of alveolar bone and teeth in the upper and lower jaw. ${ }^{1}$ It is more common in special ethnicities such as US Mexican and Indian, Japanese and Chinese, Australian Indigenous and Iranian populations. ${ }^{2-5}$ Three classic signs of this deformity include lip bulging in lateral view, lip incompetence and lip strain. For the diagnosis of this deformity, all these signs must be present.

It can be diagnosed from mixed dentition age. In comparison to normal children, those with a tendency to develop BP have a lower interincisal angle (INA), higher facial convexity and ANB. ${ }^{3}$ Adult cephalometric characteristics of patients with BP include shorter posterior cranial base, longer and more anteriorly positioned maxilla, shorter mandibular ramus and longer mandibular body, hence appropriately situated mandible, shorter upper face and posterior height, farther cephalometric horizontal planes and higher $\mathrm{H}$ angle INA $<125^{\circ}$ (normally $=131^{\circ}$ ), upper incisor to palatal plane $>115^{\circ}$ (normally $>109^{\circ}$ ) and IMPA angle $>99^{\circ}$ (normally less than $\left.93^{\circ}\right) .^{6-8}$

This deformity is associated with anterior-posterior skeletal malocclusions class I, II and III. Severe cases need orthognathic surgery.

Currently, there are two surgical methods for treatment of bimaxillary dentoalveolar protrusion class I (BPCLI): 
Segmental surgery in upper and lower jaw and complete (bimax) surgery (Lefort I in upper and ramus osteotomy in the lower jaw). These methods can both correct the occlusal correlations and the facial esthetics by affecting the facial soft tissue. ${ }^{9,10}$ Both methods are major surgeries, which impose physical, psychosocial and economic impacts on patients. If successful, it can improve the patient attractiveness and therefore, personal and social aspects of life. In contrast, if the results are not acceptable, it can lead to considerable psychosocial problems and may have several adverse consequences.

Nowadays, segmental surgery is a common practice in spite of its possible considerable complications. However, the trend is toward more use of bimax surgery as it seems more practical by the current advances in the surgery. ${ }^{11}$ In this study, we evaluated the effects of these methods on the soft tissue esthetic of face using cephalometry before and after surgery. For this purpose, upper lip thickness (ULT), nasolabial angle (NLA), nasal prominence and upper lip curvature are assessed in the patients with BPCLI, who were treated with these two methods. Results of this study can show if bimax surgery for posterior repositioning of maxilla and correction of BPCLI has priority to the currently used segmental orthognathic surgery or not.

\section{MATERIALS AND METHODS}

This study was done on 40 white-skinned Iranian patients with BPCLI who sought treatment for their deformity by referring to the Department of Oral and Maxillofacial Surgery, between 2005 and 2010. Patients were divided into two groups according to the type of treatment they were offered. In the first group, treatment includes segmental surgery for backward replacement of anterior segment of the upper and lower jaw. In the second group, treatment was bimax surgery, in which whole upper and lower jaw moved backward. Twenty patients were included in each group. Inclusion criteria were normal periodontal status, normal OVJ (overjet) and OVB (overbite), negative history of smoking, addiction and adverse habits such as bruxism, and disorders such as bone loss, diabetes, cleft lip/plate and immunodeficiency or other systemic diseases.

Before surgery, some cases may need orthodontic treatment. However, most of the patients with BPCLI have appropriate dental arches and there is no need for an interval between bracket insertion and surgery. For few other patients, we used arch bars at operation room before surgery. The plan for orthodontic preparation is different in patients receiving segmental surgery from those who are supposed to undergo bimax surgery.

In the patients treating with bimax surgery, minimal movements such as leveling, aligning and rotation corrections were performed after surgery. For patients who need orthodontic treatment before surgery, the decision to extract a tooth before or during surgery depends on the dental crowding.

\section{Model Surgery}

Casts of each patient were transferred to semiadjustable articulators with a face bow. In the bottom of casts, we placed several layers of red wax and connected them to the mounting ring of the articulator with white plaster of Paris. Then, three vertical lines in the maxillary midline, canine tip and mesiobuccal cusp of the maxillary first molar were drawn and extended onto the mandibular and mounting cast. If there was a need for upward movement of the maxilla, we drew two horizontal lines parallel to each other one on the surface of upper jaw cast and one on the mounting cast and the distance between them were documented in millimetres to use afterwards. For segmental approach, surgery was performed on the casts. After confirmation of an appropriate occlusion, segments were connected to each other by sticky wax. Then, we made lingual and palatal acrylic splints if they were designed in treatment plane. The locations of wiring were identified on the casts, and we made holes in those locations in the acrylic splints. For bimax approach, no final splint was made. Performing surgery on the upper jaw cast, we made an intermediate splint.

\section{Surgery Method ${ }^{12-16}$}

For segmental surgery, osteotomy of the upper and then lower segment was performed. No genioplasty was necessary at the time of segmental surgeries. From the three approved segmental surgeries for upper jaw, Wassmund, Wunderer and Cupar techniques, we performed Cupar technique. Incision was vestibular and thus, blood supply was from palatal vessels. Upper and lower first/second premolar teeth were extracted. From the 20 patients who underwent this surgery, we extracted first premolars in 16 and second premolars in three, and we used edentulous space in the last patient. Segmental surgery could raise anterior segment minimally (maximum of $3 \mathrm{~mm}$ ) if the patient had orthodontic appliances before surgery to eliminate the vertical step in postorthognathic arch leveling. Osteotomies were performed in a way that a minimum space of $5 \mathrm{~mm}$ from the apex of incisor teeth and a $1 \mathrm{~mm}$ thickness of bone adjacent to osteotomy sites were preserved. The osteotomy segment was fixed by screw and miniplates. We used Kole method and vestibular incision for segmental surgery in mandible (Fig. 1).

For bimax surgery, we used maxillary first technique. Lefort I and fixation, mandibular setback with BSSO (bilateral sagittal split ramus osteotomy) in 20 cases, genioplasty 


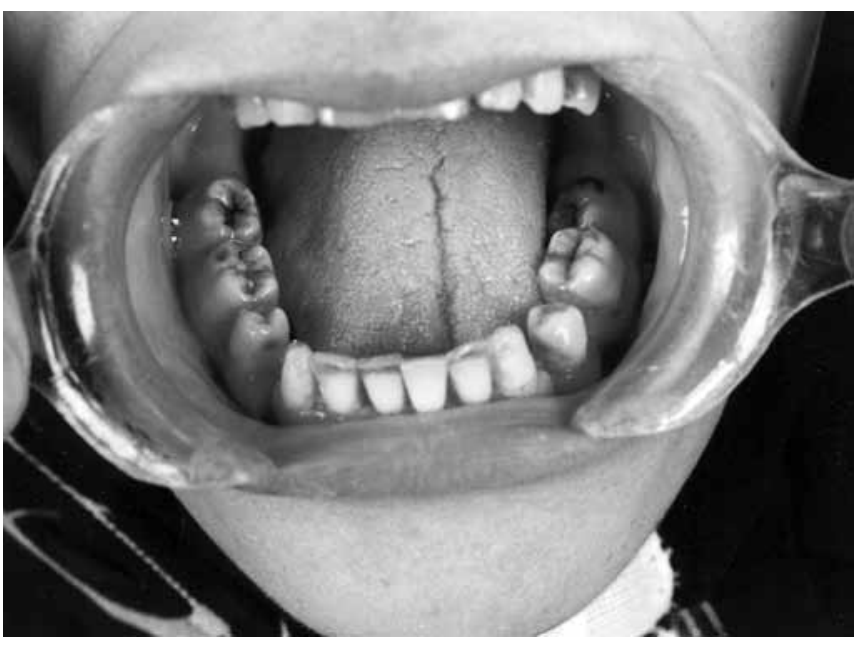

Fig. 1: Clinical picture after mandibular subapical surgery. Note remaining space in osteotomy site

and fixation was the sequence if needed. Incision of soft tissue was vestibular, and we did not use tunnel technique. The difference of our surgical method with classic Lefort I was peripterygoid canal osteotomy, fracture of pterygoid processes and send them backward or remove them and tuberosity resection in a block form, as these three more steps can harden surgery for posterior repositioning of maxilla. During surgery, we paid special attention to avoid injury to greater palatine artery. For fixation, we used four miniplates, (L shape) in pyriform and a straight plate with three holes in zygomatic buttress. After ramus osteotomy, we did rigid internal fixation (RIF) for all cases with three bicortical miniscrews in each side. For six patients, advancement genioplasty after ramus osteotomy was performed, and we moved chin 3 to $5 \mathrm{~mm}$ forward. The reason for advancement genioplasty after bimax surgery is that in contrast to segmental surgery, by this method, chin moves posteriorly after mandibular setback and there is a need for advancement genioplasty. After bimax surgery, 1 week IMF was done for all the patients and soft diet was recommended after release of IMF for 1 month.

In both methods, mucoperiosteum is detached from the bone surface, and the facial expression muscles are shortened. If these muscles are not transferred to their original place, the thickness of upper lip decreases. Therefore, after surgery, we used a 2-0 nylon stitch (Cinch technique) for maintaining the nasal alar width and a $10 \mathrm{~mm} \mathrm{V-Y} \mathrm{plasty} \mathrm{for} \mathrm{suturing} \mathrm{the}$ lip mucosa. We did not apply high bites in order to prevent narrowing of vermilion.

In contrast to changes in the bony structures, determination of alteration in soft tissue after surgery is still difficult. There are several modulating factors in the assessment of soft tissue changes. After correction of BPCLI deformity by segmental or bimax surgeries, the important factors include the technique of surgery (e.g. incision method), adaptation of soft tissue, patient growth, lip thickness, tone, interlips distance, OVJ, volume of fat and muscular tissue and postsurgical edema. To reduce the effect of incision method, vestibular incision was applied in both groups. For lowering the impact of other factors on the results of our study, patients were evaluated after 6 months. After this time, edema is cleared and tissue distribution would re-establish.

Before and 6 months after surgery, we performed lateral cephalometric radiographies with patients in NHP centric occlusal resting positions. Radiographies were placed in negatoscope with the lateral views of face being in the right side. To the attached acetate tracing papers, anatomic indices of soft and hard tissues were drawn (hand analysis). The soft tissue indices include pronasal (PRN, the top point of nasal tip), subnasal (SN, cross point of nasal septum and upper lip skin in the sagittal plane), labral superius (LS, the most anterior point of the upper lip), sublabial sulcus (SLS, the midline deepest point of concavity between LS and SN), pogonion ( $\mathrm{PG}$, the most anterior point in the contour of chin soft tissue). Hard tissue indices include A-point (the most backward point of anterior surface of the maxilla), IA (the most prominent point in the labial surface of upper incisor), Po (the top point on the anatomic external acoustic duct) and Or (the lowest point on the inferior border of orbit). We designed three planes: Frankfort horizontal plane (FHP, line connecting Co to Or), Holdaway line (H-Line, line abutting cutaneous pogonion and upper lip) and perpendicular line (from LS to Frankfort plane). These indices were determined to assess the soft tissue variables we used in our study to evaluate the outcome of segmental and bimax surgeries regarding changes in the soft tissues. For this purpose, we measured ULT (distance between LS and IA), nose prominence (NP, distance between nasal tip and the perpendicular line from upper lip vermilion on FHP), subsulcus depth (SSD, distance of SLS from this perpendicular line), SN to $\mathrm{H}$ line distance and finally, NLA. To measure NLA, we should first determine PCMM, the most posterior point on the lower border of the nose from which it turns downward to reach lip philtrum. In fact, NLA was defined as the angle at PCMM between a line tangent to the lower border of the nose and a line from PCMM to LS (Figs 2A to E).

\section{DATA ANALYSIS}

Data was analyzed by the statistical tests of SPSS software. Paired t-test was used to determine the changes in soft tissue parameters before and after surgeries. p-value $<0.005$ considered as significant difference. Kolmogorov-Smirnov test was used to assess the normality 


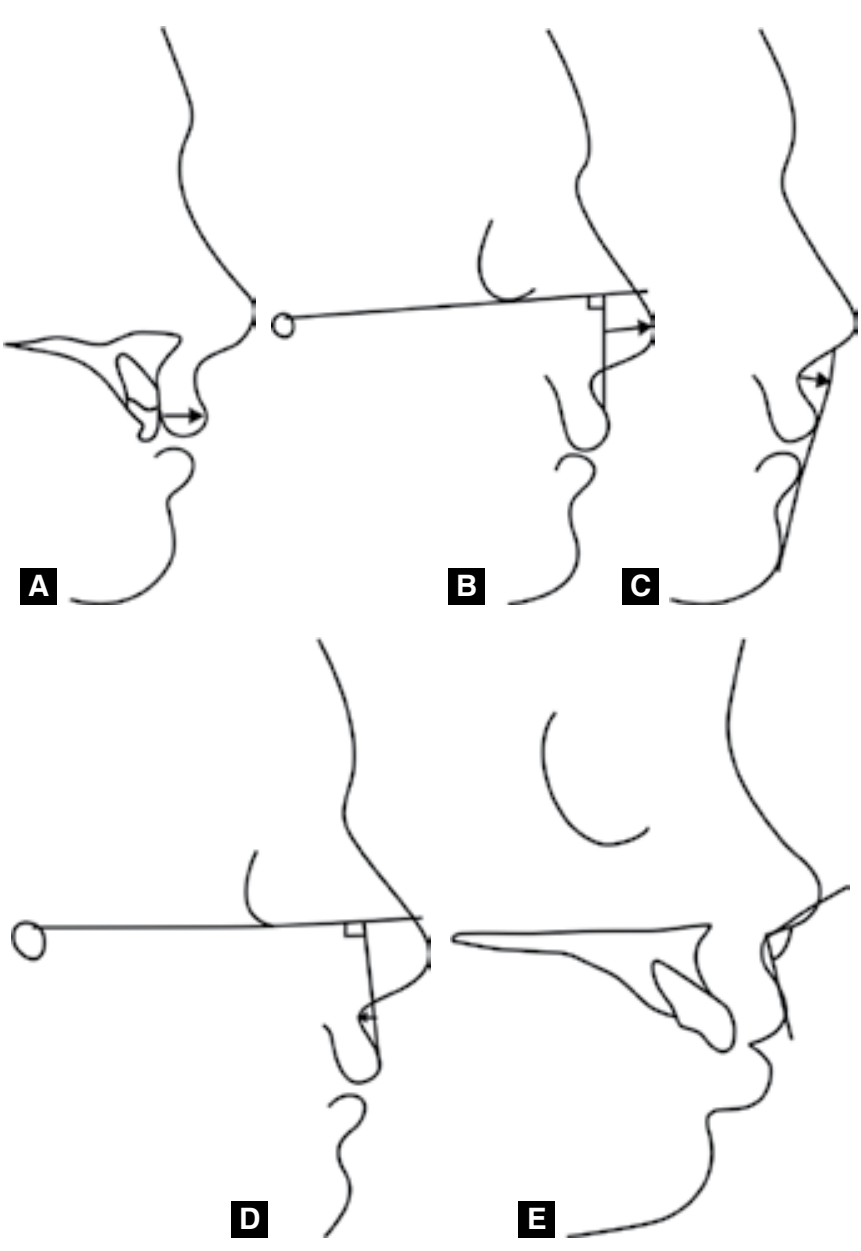

Figs 2A to E: Soft tissue parameters that measured in this study. (A) ULT: Upper lip thickness, (B) NP: Nose prominence, (C) SSD: Subsulcus depth, (D) SN to H line, (E) NLA: Nasolabial angle

of data distribution for each variable. For those factors that had a normal distribution, we used Student t-test for analysis of data. Wilcoxon test was the statistical test that we applied for variables without normal distribution.

\section{RESULTS}

In our study, $65 \%$ of patients were female and the mean of age was 27 (17-39) years old. The mean of SNA, SNB, ANB and INA in our patients were $81.7 \pm 2.9,78.8 \pm 2.8$, $4.50 \pm 1.4$ and $120 \pm 8.7$, respectively. Table 1 shows the soft tissue parameters before and after segmental and bimax surgeries for treatment of BPCLI. Results of KolmogorovSmirnov test for assessment of the normality of data distribution for each variable are given in Table 2. According to the results of this test, all the variables except SSD had a normal distribution. Therefore, all variables except SSD were analyzed with t-test to compare the results of two methods of surgery.

Differences in the values of NP, NLA, SN to $\mathrm{H}$ line distance and ULT before and after segmental and bimax surgeries are given in Table 3 . As it could be observed, the differences in values of the variables between before and after surgery were significant. After surgeries, ULT and the $\mathrm{SN}$ to $\mathrm{H}$ line distance reduced significantly, and NLA became corrected to its normal range (90-1,100).

Wilcoxon test showed that the upper lip curvature after segmental surgery had a significant difference from the

\begin{tabular}{|c|c|c|c|}
\hline Variable & & Z-test & $p$-value \\
\hline \multirow[t]{2}{*}{ NLA } & Before surgery & 1.032 & 0.237 \\
\hline & After surgery & 0.442 & 0.99 \\
\hline \multirow[t]{2}{*}{$\mathrm{SN}$ to $\mathrm{H}$-Line } & Before surgery & 0.793 & 0.555 \\
\hline & After surgery & 1.091 & 0.185 \\
\hline \multirow[t]{2}{*}{ ULT } & Before surgery & 0.982 & 0.29 \\
\hline & After surgery & 1.286 & 0.073 \\
\hline \multirow[t]{2}{*}{ NP } & Before surgery & 0.582 & 0.887 \\
\hline & After surgery & 1.075 & 0.198 \\
\hline \multirow[t]{2}{*}{ SSD } & Before surgery & 1.241 & 0.092 \\
\hline & After surgery & 1.391 & 0.042 \\
\hline
\end{tabular}

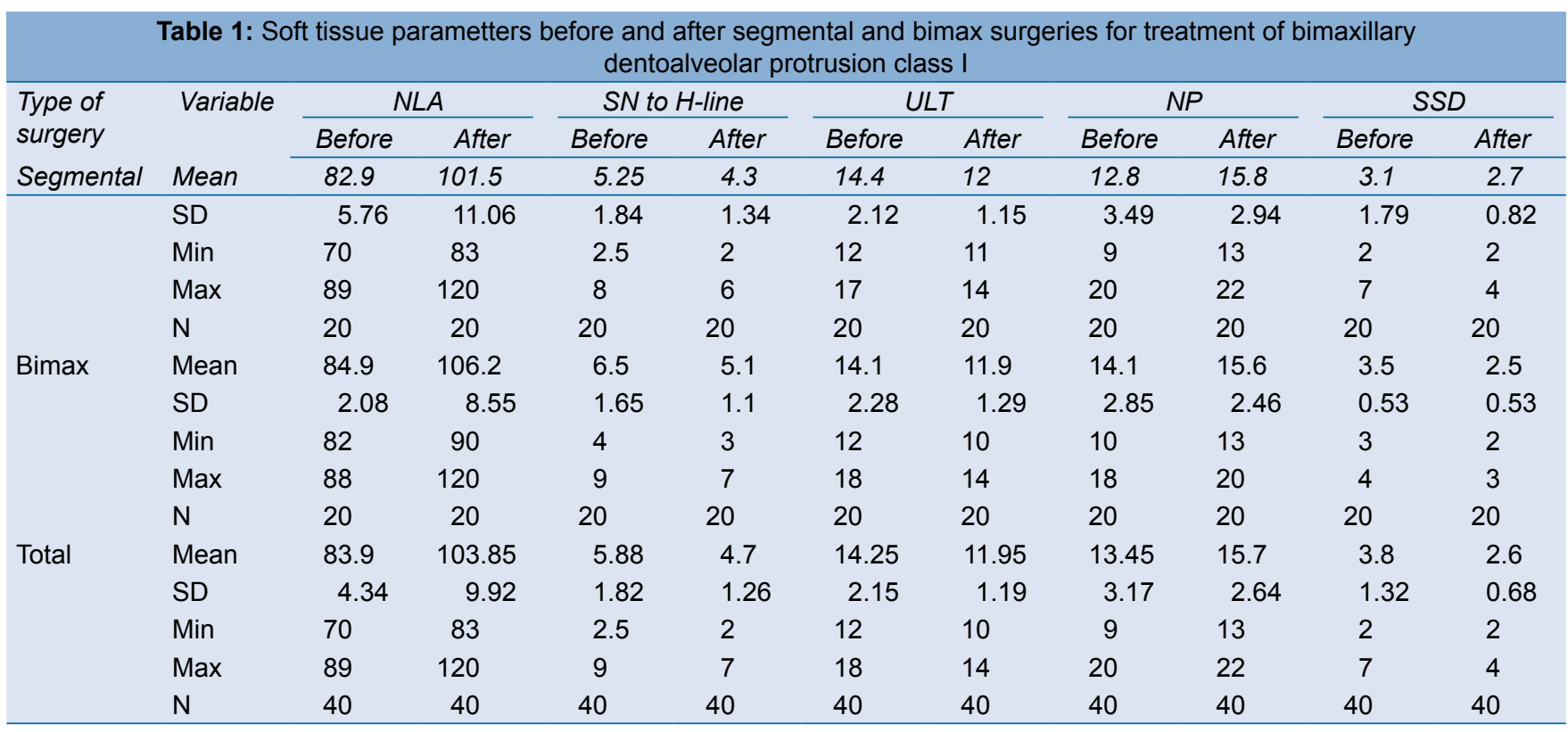


Effects of Bimax and Segmental Surgeries for Correction of Bimaxillary Dentoalveolar Protrusion Class I on Soft

\begin{tabular}{|c|c|c|c|c|c|}
\hline Type of surgery & Variables & Mean difference & Variance & T-test value & $p$-value \\
\hline \multirow[t]{4}{*}{ Segmental } & NLA & -18.6 & 7.96 & -7.647 & 0 \\
\hline & $\begin{array}{l}\mathrm{SN} \text { to } \mathrm{H} \text {-line } \\
\text { distance }\end{array}$ & 0.95 & 0.685 & 4.385 & 0.002 \\
\hline & ULT & 2.4 & 1.074 & 7.06 & 0 \\
\hline & NP & -3 & 0.816 & -1.161 & 0.002 \\
\hline \multirow[t]{4}{*}{ Bimax } & NLA & -21.3 & 7.364 & -9.146 & 0 \\
\hline & $\begin{array}{l}\mathrm{SN} \text { to } \mathrm{H} \text {-line } \\
\text { distance }\end{array}$ & 1.4 & 0.843 & 5.25 & 0.001 \\
\hline & ULT & 2.2 & 1.316 & 5.284 & 0.001 \\
\hline & $\mathrm{NP}$ & -1.5 & 0.849 & -5.582 & 0 \\
\hline \multirow[t]{4}{*}{ Total } & NLA & -19.95 & 7.458 & -11.962 & 0 \\
\hline & $\begin{array}{l}\mathrm{SN} \text { to } \mathrm{H} \text {-line } \\
\text { distance }\end{array}$ & 1.175 & 0.782 & 6.714 & 0 \\
\hline & ULT & 2.3 & 1.174 & 8.759 & 0 \\
\hline & NP & -2.25 & 1.118 & -9 & 0 \\
\hline
\end{tabular}

Table 4: Wilcoxon test for analysis of upper lip curvature (subsulcus depth: SSD) before and after surgery

\begin{tabular}{lccc} 
Variable & $\begin{array}{c}\text { Total } \\
(n=40)\end{array}$ & $\begin{array}{c}\text { Segmental surgery } \\
(n=20)\end{array}$ & $\begin{array}{c}\text { Bimax surgery } \\
(n=20)\end{array}$ \\
\hline Z-test & -3.35 & -2.558 & -2.271 \\
p-value & 0.001 & 0.011 & 0.23 \\
\hline
\end{tabular}

curvature before segmental surgery. However, the reduction after bimax surgery was not significant (Table 4).

\section{DISCUSSION}

Any surgery performed on the face is highly important as it can affect not only the appearance of patients but also significantly influencing on the psychosocial aspects of the patient life. ${ }^{11}$ In those who need facial surgery, the success of this surgery can increase the chance of personal and social achievements. Facial surgery is performed for several purposes, and one of the most important reasons is for correction of congenital malformations. Bimax and segmental surgeries are the main surgical techniques for correction of BPCLI. Like other surgical techniques, they have advantages and disadvantages that must be taken into consideration when a decision is to be made for a patient. This study was performed to assess the soft tissue outcomes of bimax and segmental surgeries for BPCLI deformity. This study helps to decide which surgery to choose as it discusses the features and correction rate of soft tissue parameters before and after each surgery.

It is worth knowing that segmental surgery was introduced far before complete maxillary osteotomies. It is thought that segmental surgery improves occlusion in the cost of facial esthetic. By the introduction of Lefort surgeries and improvement of wound healing techniques, it seems that there is a reduction in the number of segmental surgeries these days. Another reason for this trend is that bone osteotomy of segmental orthognathic surgery are adjacent to periodontal tissues and therefore, the dental/periodontal injuries during interdental osteotomies are highly probable. ${ }^{17}$
In a study the probability of periodontal bone loss in the interdental osteotomies regions was reported as high as $18.4 \% .{ }^{18}$ However, the disadvantages are not only limited to segmental surgery and certainly bimax surgery has its own disadvantages as well.

This study showed that in contrast to segmental surgery, bimax surgery was not associated with a significant change in the upper lip curvature. On the other hand, with segmental surgery, upper lip curvature (SSD) reduced and reached to the normal value $(3 \mathrm{~mm})$. It was shown that the more SSD is near to this value, the more patient become attractive. However, it should be noticed that SSD must not be reduced to lower than $1.5 \mathrm{~mm}$ after surgery; otherwise it can lead to flattening of upper lip and decrease in the person's attractiveness. Data of this study showed that these surgeries could maintain the upper lip curvature in the normal range.

Furthermore, these surgeries could increase the nose prominence. There is no normal range for this parameter, as it depends on the patient's opinion and must be assessed individually. However, because the nose might look more prominent after surgery, there might be a need for a future rhinoplasty, and this must be explained to the patients and their parents before surgery. Another important point in the results of this study is that both surgeries would reduce ULT and thus make the patient look older.

NLA is an important factor in making decision for orthognathic surgery and orthodontic treatments. This study showed that we could correct NLA by bimax and segmental surgeries. The normal range of NLA is $105.8 \pm 9$ for male and $110.7 \pm 10.9$ for female older than 18 years old. However, for interpretation of NLA before surgery, the nose contour is very important as NLA might be normal despite clinical presence of BP. The reason of normal NLA in this case might be the upward rotation of the nasal tip. ${ }^{19}$ Previous studies showed that segmental surgery can correct NLA. For instance, segmental surgery could increase NLA from $95 \pm 11.6$ to $103.9 \pm 7.5$ in a study by Nadkarni in Indians, ${ }^{20}$ $77.4 \pm 3.6$ to $89.6 \pm 3.9$ in Lew's study in China, ${ }^{21}$ and 
$91.8 \pm 11.3$ to $104 \pm 7.6$ in a Kim investigation in Korea. ${ }^{5}$ Correction of NLA was also possible by orthodontic treatment. Kusnoto showed that NLA increased from 97 to 104.8 by orthodontic treatment in Indonesian population. ${ }^{22}$ Another study in China also showed an increase in NLA from 86.6 to 97.1 by orthodontic treatment in BPCLI patients. ${ }^{23}$ It should be noticed that NLA has the correlation with the morphology of nose and upper lip. It seems that the effect of retraction of upper incisors with orthodontic treatment or surgery on NLA is more associated with the position of upper lip vermilion rather than the nose position $(90 \%$ vs $10 \%) .{ }^{24}$ Bimax surgery could increase NLA more than segmental surgery although this increase is not statistically significant. The reason for a better correction of NLA by bimax surgery is that this technique can alter the angle between mandibular plane and Frankfort horizontal plane. It is confirmed that patients with an open mandibular plane angle and simultaneous maxillary protrusion have more clinical problems and an augmentation in the posterior height of face and MPA (mandibular plane angle) during treatment can increase NLA. Even mandibular setback surgery alone in patients with prognathism can raise NLA. $^{25}$

In our study, INA was in the normal range. It seems that interincisal angle has a correlation with the ethnicity, as the results of other two studies show that in Chinese and Caucasian patients with BPCLI deformity, the value of this angle was considerably lower than our results, 102.7 and 115.2 , respectively. ${ }^{6,23}$

To sum up, assessment of soft tissue parameters before and after segmental and bimax surgeries showed that both techniques could clinically improve the parameters. The most outstanding improvement was about NLA. While data analysis should statistical significant differences between the values of these parameters before and after surgery, bimax surgery could not decrease upper lip curvature in comparison to segmental surgery. Because NLA is one of the main factors in the correction of BP deformity and increase in NLA can improve the facial beauty, we suggest bimax surgery more for those patients with BP that needs correction of NLA.

\section{CONCLUSION}

Considering the results of this study and the problems of segmental surgery, we highly recommend bimax surgery for treatment of BPCLI and also suggest that for an ideal outcome, it might be much better to add advancement genioplasty to the treatment plan of bimax surgery. This procedure strongly recommended for BPCLI patients with previous unsuccessful orthodontic attempts with teeth extractions and vertical maxillary excess. This procedure need not tooth extraction so space closure especially in lower jaw is not a concern if the patient is not decided to do orthodontic treatment. However, the effects of posterior repositioning of maxilla on the upper airway and Eustachian tube function must be assessed in further investigations.

\section{REFERENCES}

1. Hashimoto T, Kuroda S, Kamioka H, Mishima K, Sugahara T, Takano-Yamamoto T. Bimaxillary protrusion with masseter muscle hypertrophy treated with titanium screw anchorage and masseter surgical reduction. Am J Orthod Dentofacial Orthop 2009;135(4):536-548.

2. Lamberton CM, Reichart PA, Triratananimit P. Bimaxillary protrusion as a pathologic problem in the Thai. Am J Orthod 1980;77(3):320-329.

3. Tsai HH. Cephalometric characteristics of bimaxillary dentoalveolar protrusion in early mixed dentition. J Clin Pediatr Dent 2002;26(4):363-370.

4. Hajighadimi M, Dougherty HL, Garakani F. Cephalometric evaluation of Iranian children and its comparison with Tweed's and Steiner's standards. Am J Orthod 1981;79(2):192-197.

5. Kim JR, Son WS, Lee SG. A retrospective analysis of 20 surgically corrected bimaxillary protrusion patients. Int $\mathrm{J}$ Adult Orthodon Orthognath Surg 2002;17(1):23-27.

6. Keating PJ. Bimaxillary protrusion in the Caucasian: a cephalometric study of the morphological features. Br J Orthod 1985;12(4):193-201.

7. Jacobs JD, Bell WH. Combined surgical and orthodontic treatment of bimaxillary protrusion. Am J Orthod 1983;83(4):321-333.

8. Langberg BJ, Todd A. Treatment of a class I malocclusion with severe bimaxillary protrusion. Am J Orthod Dentofacial Orthop 2004;126(6):739-746.

9. O'Reilly MT. Integumental profile changes after surgical orthodontic correction of bimaxillary dentoalveolar protrusion in black patients. Am J Orthod Dentofacial Orthop 1989;96(3): 242-248.

10. Connor AM, Moshiri F. Orthognathic surgery norms for American black patients. Am J Orthod 1985;87(2):119-134.

11. Jayaratne YS, Zwahlen RA, Lo J, Cheung LK. Facial soft tissue response to anterior segmental osteotomies: a systematic review. Int J Oral Maxillofac Surg 2010;39(11):1050-1588.

12. Tomlak DJ, Piecuch JF, Weinstein S. Morphologic analysis of upper lip area following maxillary osteotomy via the tunneling approach. Am J Orthod 1984;85(6):488-493.

13. Collins PC, Epker BN. The alar base cinch: a technique for prevention of alar base flaring secondary to maxillary surgery. Oral Surg Oral Med Oral Pathol 1982;53(6):549-553.

14. Cupar I. Surgical treatment of alterations in form and position of the maxilla. Osterr Z Stomatol 1954;51(11):565-577.

15. Kole H. Surgical operations on the alveolar ridge to correct occlusal abnormalities. Oral Surg Oral Med Oral Pathol 1959;12(5):515-529.

16. Schouman T, Baralle MM, Ferri J. Facial morphology changes after total maxillary setback osteotomy. J Oral Maxillofac Surg 2010;68(7):1504-1511.

17. Hutchinson $D$, MacGregor AJ. Tooth survival following various methods of subapical osteotomy. Int J Oral Surg 1972;1(2): 81-86. 
18. Tueki K, Marukawa K, Shimada M, Alam S, Nakagawa $\mathrm{K}$, Yamamoto E. The prevention of periodontal bone loss at the osteotomy site after anterior segmental and dentoosseous osteotomy. J Oral Maxillofac Surg 2006;64(10): 1526-1531.

19. Fitzgerald JP, Nanda RS, Currier GF. An evaluation of the nasolabial angle and the relative inclinations of the nose and upper lip. Am J Orthod Dentofacial Orthop 1992;102(4): 328-334.

20. Nadkarni PG. Soft tissue profile changes associated with orthognathic surgery for bimaxillary protrusion. J Oral Maxillofac Surg 1986;44(11):851-854.

21. Lew KK, Loh FC, Yeo JF, Loh HS. Profile changes following anterior subapical osteotomy in Chinese adults with bimaxillary protrusion. Int J Adult Orthodon Orthognath Surg 1989;4(3): 189-196.

22. Kusnoto J, Kusnoto $\mathrm{H}$. The effect of anterior tooth retraction on lip position of orthodontically treated adult Indonesians. Am J Orthod Dentofacial Orthop 2001;120(3):304-307.

23. Tan TJ. Profile changes following orthodontic correction of bimaxillary protrusion with a preadjusted edgewise appliance. Int J Adult Orthodon Orthognath Surg 1996;11(3):239-251.
24. Lo FD, Hunter WS. Changes in nasolabial angle related to maxillary incisor retraction. Am J Orthod 1982;82(5):384391.

25. Mobarak KA, Krogstad O, Espeland L, Lyberg T. Factors influencing the predictability of soft tissue profile changes following mandibular setback surgery. Angle Orthod 2001;71(3):216-227.

\section{ABOUT THE AUTHORS}

\section{Amin Rahpeyma}

Associate Professor, Department of Oral and Maxillofacial Surgery Oral and Maxillofacial Diseases Research Center, School of Dentistry Mashhad University of Medical Sciences, Mashhad, Iran

\section{Saeedeh Khajehahmadi (Corresponding Author)}

Assistant Professor, Department of Oral and Maxillofacial Pathology Dental Research Center, School of Dentistry, Mashhad University of Medical Sciences, Mashhad, Iran, e-mail: khajehahmadis@mums. ac.ir, saeedeh.ahmady@yahoo.com 